\section{Utility of optic pathway glioma screening in young children with neurofibromatosis type I: questions generated by a clinical audit}

RF Pilling', IC Lloyd' and S Huson²

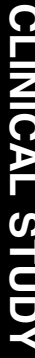

Eye (2010) 24, 1603-1605; doi:10.1038/eye.2010.99; published online 6 August 2010

Keywords: neurofibromatosis; optic pathway glioma; optic nerve glioma; screening

Introduction

Neurofibromatosis type I (NFI) is a phakomatosis that affects around 1 per 3000 live births. It is a multi-system disorder with hamartomas or neurofibromas affecting most commonly neural tissue. About $15 \%$ of children with NFI develop optic pathway glioma (OPG). ${ }^{1,2}$ The natural history of these tumours is still poorly understood. About two thirds do not progress or become visually significant. ${ }^{2}$ They do not metastasise but may affect hypothalamic function. OPG classically present before age 6, but rarely is the child symptomatic of their visual loss. ${ }^{3}$ The Neurofibromatosis UK society recommend annual ophthalmic screening to identify those children who may have OPG affecting vision and refer for investigation and treatment as necessary. ${ }^{3}$ The recommended protocol includes vision, pupil, colour vision, visual field, and optic disc assessment.

We planned an audit to assess how well our department complied with these recommendations and the number of children who had an OPG detected as a result of screening.

\section{Materials and methods}

The primary aim of the audit was to elicit if ophthalmology screening practice of children
${ }^{1}$ Manchester Royal Eye Hospital, Oxford Road, Manchester, UK

${ }^{2}$ Department of Clinical Genetics, St Mary's Hospital, Oxford Road, Manchester, UK

Correspondence: RF Pilling, Manchester Royal Eye Hospital,

Oxford Road,

Manchester M13 9WL, UK Tel: + 44 (0)161 276 5565; Fax: + 44 (0)161 2765531 E-mail: Rachel.Pilling@ cmft.nhs.uk

Received: 25 February 2010 Accepted in revised form: 8 June 2010 Published online: 6 August 2010 
Table 1 Criteria and standards chosen for audit

\begin{tabular}{ll}
\hline Criterion & $\begin{array}{c}\text { Standard } \\
(\%)\end{array}$ \\
\hline 1 Children with NFI should receive annual screening & 100 \\
in the eye clinic for optic pathway glioma (OPG) & \\
from the age of 0-7 years (1-3) & 100 \\
2 Children should undergo visual acuity testing (1-3) & 100 \\
3 Children should have pupillary reactions tested & 100 \\
4 Children should have optic disc assessment & 100 \\
5 Children should have colour vision assessment & \\
once mature enough & 100 \\
6 & $\begin{array}{l}\text { Children should have visual field assessment once } \\
\text { mature enough }\end{array}$ \\
\hline
\end{tabular}

Table 2 Data collected from clinical notes and corresponding criterion answered

\begin{tabular}{lc}
\hline Data & Criterion tested \\
\hline Age at diagnosis of NF & 1 \\
How many screening visits would we expect & 1 \\
them to have had? & 1 \\
How many screening visits have they had? & 3 \\
Age at first ophthalmological examination & \\
Ocular findings at first screening & 2 \\
Vision & 3 \\
Pupillary reaction (RAPD) & 4 \\
Optic nerve appearance & 5 \\
Colour vision & 6 \\
Visual fields & \\
\hline
\end{tabular}

with NFI for OPG meets current guidelines. Secondary aims of the audit were to document the age at which tests of visual function are useful in the diagnosis and screening of OPG, and to document the contribution eye screening has made to the diagnosis of OPG. The audit was reviewed and approved by the Central Manchester Foundation Trust Audit Board.

Six criteria were chosen based on the Neurofibromatosis UK guidelines for screening for OPG in children with $\mathrm{NFI}^{3}$ (Table 1). As there are no published standards, we elected to choose a $100 \%$ standard for all criteria.

We undertook a retrospective data extraction from clinical notes. We included all children born on or after 1 January 2000 attending the ophthalmology clinic for OPG screening. These children were identified from the Eye Clinic Database and cross-referenced against the genetic department database of known neurofibromatosis cases. Data were collected as shown in Table 2.

\section{Results}

A total of 37 children were identified from the register. They were confirmed as attending clinic for
Table 3 Summary of results

\begin{tabular}{lcc}
\hline Criterion & Standard (\%) & Achieved (\%) \\
\hline 1 Annual screening & 100 & 43 \\
2 Visual acuity & 100 & 100 \\
3 Pupil/RAPD & 100 & 84 \\
4 Optic disc assessment & 100 & 100 \\
5 Colour vision & 100 & No children \\
& & mature enough \\
6 Visual fields & 100 & No children \\
& & mature enough \\
\hline
\end{tabular}

screening for OPG. A summary of the results is shown in Table 3.

Of these, 16 achieved standard 1 (43\%): that is, they were screened annually from diagnosis to age 7 . Of the 21 who did not comply with the standard, 13 had failed to attend clinic multiple times ('DNA'), 2 had their appointment cancelled by the hospital, and 1 had a combination of DNA and cancellations. Some children had not ever been screened as a result, whereas some had not been seen at the required number of screening appointments.

Five children were seen more often than recommended. This occurred most commonly in those children under age 2 who were screened every 6 months as they were thought to be at highest risk of developing an OPG and/or it was not been possible to measure uniocular visual acuity. One child was monitored for a plexiform neurofibroma affecting her lid, and one for an esotropia. As a result, these children were seen more frequently in the eye clinic.

The mean age at diagnosis of NFI was 23 months, the mean age at first screen was 29 months, and on average children had three screening episodes. Visual acuity assessment was possible on all patients. A 'both eye only' vision was achieved in seven children at first visit. Subsequent visits allowed uniocular vision assessment.

An RAPD was not performed in six cases: most often these were first visits and were dilated by the orthoptist for refraction before seeing the doctor. Discs were assessable and normal in all except two: one with astrocytoma, the other with pale discs.

Colour and visual fields were attempted in only two children; age 6 and 7. The tests were abandoned due to poor cooperation.

\section{Discussion}

The primary aim of this audit was to establish to what extent children undergo ophthalmology screening as laid out in the guidelines: are we following best practice.

We have shown that less than half of children achieve the 
standard, mainly due to appointment non-attendance. This could be remedied by providing carers with more information about why eye screening is necessary or providing a joint clinic with children seen during one of their other hospital visits.

The ophthalmic assessment of the children complies with current guidelines. It may be that further education is required to ensure that children are not dilated before having pupillary reactions tested, as is the common practice in many other new patient referrals to eye clinic. The presence of an RAPD is a subtle and early sign of optic nerve dysfunction and an essential part of the screening protocol.

It is interesting to note how infrequently children were thought to be mature enough to perform visual fields and colour vision. The usefulness of other measures of visual function such as visual fields or colour vision is unknown: these tests are difficult to perform in the target age group and, in the absence of reduced vision, are unlikely to contribute to decision-making. It is our experience that testing colour vision and visual fields in children in the high-risk age group is technically very demanding and may not be the most appropriate use of orthoptic resources.

The secondary aim was to evaluate the effectiveness of screening to detect clinically relevant OPG: indeed does it 'screen' for the condition. A total of 126 screening episodes were carried out during the period studied (approximately 7 years). No OPG was detected as a result of these screening episodes. Because children were screened only once annually, because the growth of tumours is unpredictable, and because the visual function of children in this age group is variable, it could be argued that vision screening once a year may not contribute to the diagnosis, leading to treatment, of OPG.

Summary

What was known before

- National guidelines exist for the screening of children with NF1 for OPG.

- Most children diagnosed with OPG associated with NF1 are not diagnosed through ophthalmic screening.

- Visual fields and colour vision are difficult to assess formally in young children.

What this study adds

- There is poor adherence to the screening protocol mainly due to non-attendance.

- No OPG was diagnosed during 126 screening episodes (approximately 7 years).

- Visual fields and colour vision were not attempted or successful in any children in the high-risk age group and their utility in a screening guideline could be questioned.

\section{Conflict of interest}

The authors declare no conflict of interest.

\section{References}

1 Listernick RM, Louis DN, Packer RJ, Futmann DH. Optic pathway gliomas in children with NFI: consensus statement from the NFI Optic Pathway Glioma Task Force. Ann Neurol 1997; 41: 143-149.

2 Listernick MD, Ferner RE, Liu GT, Gutmann EH. Optic pathway gliomas in NFI: controversies and recommendations. Ann Neurol 2007; 61: 189-198.

3 Ferner RE, Huson SM, Thomas N, Moss C, Wilshaw H, Evans DG et al. Guidelines for the diagnosis and management of individuals with NFI. J Med Genet 2007; 44: 81-88. 